\title{
Progress toward a Soft X-ray Polarimeter
}

\author{
Herman L. Marshall ${ }^{a}$, Norbert S. Schulz ${ }^{a}$, Brian Remlinger ${ }^{a}$, Eric S. Gentry ${ }^{a}$, David L. Windt ${ }^{b}$ \\ , Eric M. Gullikson ${ }^{c}$ \\ ${ }^{a}$ MIT Kavli Institute, Cambridge, MA, USA 02139 \\ ${ }^{b}$ Reflective X-ray Optics, 1361 Amsterdam Ave, Suite 3B, New York, NY, USA 10027 \\ ${ }^{c}$ Lawrence Berkeley National Lab, 1 Cyclotron Rd., Bldg. 2R0400, Berkeley, CA, USA 94720
}

\begin{abstract}
We are developing instrumentation for a telescope design capable of measuring linear X-ray polarization over a broad-band using conventional spectroscopic optics. Multilayer-coated mirrors are key to this approach, being used as Bragg reflectors at the Brewster angle. By laterally grading the multilayer mirrors and matching to the dispersion of a spectrometer, one may take advantage of high multilayer reflectivities and achieve modulation factors over $50 \%$ over the entire $0.2-0.8 \mathrm{keV}$ band. We present progress on laboratory work to demonstrate the capabilities of an existing laterally graded multilayer coated mirror pair. We also present plans for a suborbital rocket experiment designed to detect a polarization level of $12-17 \%$ for an active galactic nucleus in the $0.1-1.0$ $\mathrm{keV}$ band.
\end{abstract}

Keywords: X-ray, polarimeter, astronomy, multilayer, mirror, grating

\section{INTRODUCTION}

We continue our investigation and laboratory work to develop a soft X-ray polarimeter based on Bragg reflection from multilayer-coated optics. Marshall (2007-1) described a method using transmission gratings to disperse the incoming X-rays so that the dispersion is matched to laterally graded multilayer (ML) coated reflectors. An extension of this approach was suggested by Marshall (20082 $)$ that can be used with larger missions such as the AXSIO or AEGIS. Some potential scientific investigations that would be possible with a soft X-ray polarimeter were described earlier and include testing the synchrotron nature of quasar jet emission and models of neutron star atmospheres $3 \frac{3.4}{4}$

The laboratory work was initiated in order to test prototype optics for eventual use in a flight design. The work started with simple measurements after recommissioning the 17-m X-ray beamline at MIT's building NE80. Murphy et al. $\left(2010^{5}\right.$ ) showed early results and described most of the system in detail. Here, we show results after improving alignment, resulting in a uniform, monochromatic beam, and the first successful polarization test. We also describe work to develop new ML coatings and laterally graded ML coated mirrors (LGMLs) and the next steps in our lab development.

A description of a design for a suborbital rocket flight is given in $\$ 3$. The experiment's minimum detectable polarization (MDP) is expected to be $6.5 \%$ when observing a bright blazar such as Mk 421 .

\section{THE MIT POLARIMETRY BEAMLINE}

We have recently recommissioned the X-ray grating evaluation facility (X-GEF), a $17 \mathrm{~m}$ beamline that was developed for testing transmission gratings fabricated at MIT for the Chandra project. ${ }^{6}$ The project development is proceeding in four distinct phases, of which two have been completed. In Phase I, we set up the polarized $\mathrm{X}$-ray source at one energy $(0.525 \mathrm{keV})$ and aligned it so that the beam was uniform at the detector and its intensity did not vary significantly with rotation angle. In Phase II, we added a ML coated mirror to the detector end of the system and reoriented the detector to face $90^{\circ}$ to the beamline and demonstrated that the source produced nearly $100 \%$ polarized X-rays.

Further author information: (Send correspondence to H.L.M.)

H.L.M.: hermanm@space.mit.edu, Telephone: 16172538573 

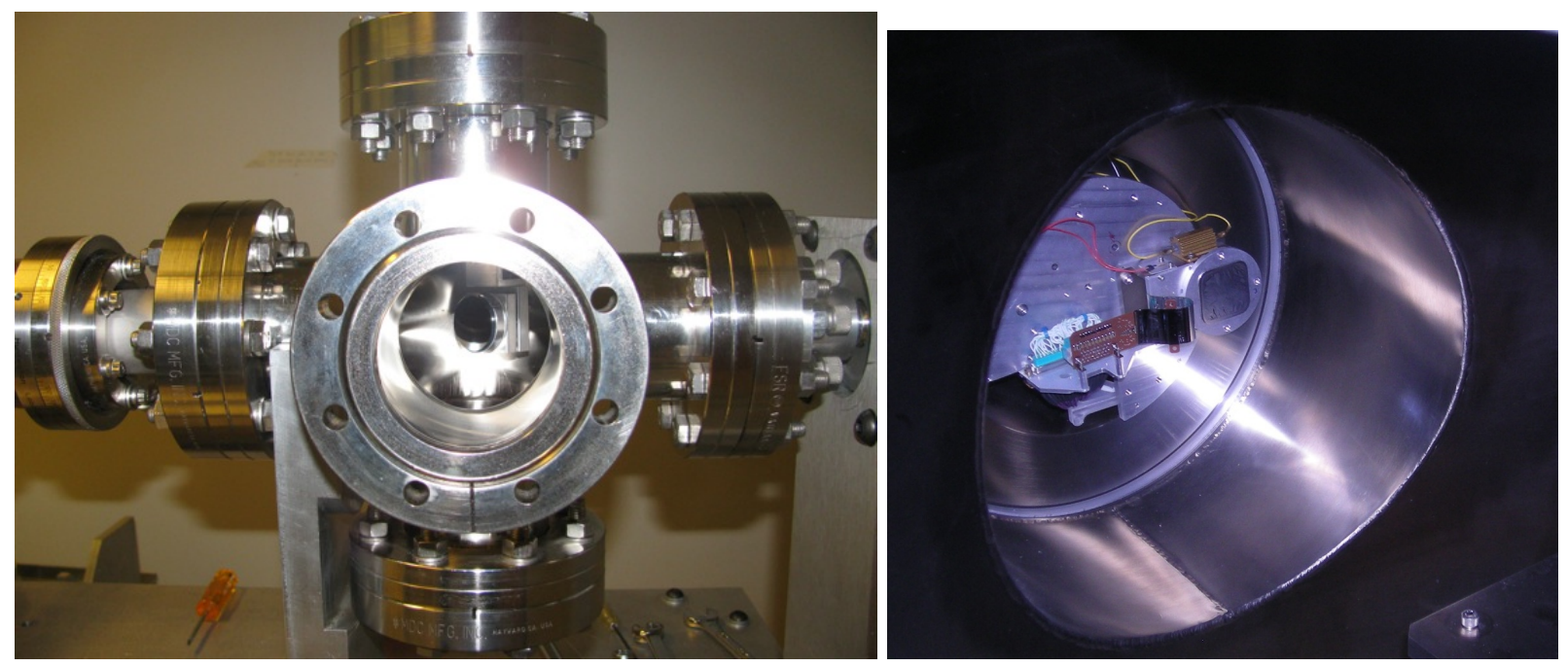

Figure 1. Hardware in the polarimetry beamline for Phases I and II. Left: A single-period ML coated mirror is mounted at $45^{\circ}$ to the beamline (at left), as viewed from the flange to which the X-ray source is mounted. The mirror is about $25 \mathrm{~mm}$ in diameter and is attached to an $\mathrm{Al}$ mount with adjustment screws to rotate it about two axes: vertical and horizontal. The rotatable vacuum flange is shown at left and the motor that rotates the source and mirror chambers is attached at right. The source rotation phase angle is defined to be zero at the orientation shown in the picture and $90^{\circ}$ when the X-ray source has been rotated to be above the beamline. Right: The CCD detector, mounted on a 200 mm flange, as viewed from the detector chamber, about $17 \mathrm{~m}$ from the X-ray source.

We are now in Phase III, where we are replacing the source ML coated mirror with a LGML in order to polarize a range of input emission lines from 0.2 to $0.8 \mathrm{keV}$. In Phase IV, we will insert a diffraction grating in order to disperse the polarized input onto an LGML in the detector chamber. At the end of Phase IV, we will be ready to test LGMLs with improved reflectivities and with larger ML coating periods in order to demonstrate that they can be used in a flight system, such as the one described in $\$ 3$.

\subsection{Polarimetry Beamline Phase I}

With MKI technology development funding, we adapted the source to produce polarized X-rays at the O-K $\alpha$ line $(0.525 \mathrm{keV}) \stackrel{5}{5}$ A five-way chamber was added to house the Polarized Source MultiLayer (PSML) mirror (see Fig. 11). The mirror and a twin were provided by Reflective X-ray Optics (RXO), with a coating consisting of 200 layers of $5.04 \AA$ of $\mathrm{W}$ alternating with $11.76 \AA$ of $\mathrm{B}_{4} \mathrm{C}$. The wavelength, $\lambda$, of the Bragg peak for a periodic ML coating is given by $\lambda=2 D \sin \theta$, where $D=16.80 \AA$ is the average layer thickness, and $\theta=45^{\circ}$ is the graze angle (measured from the surface). The source is mounted to this chamber at $90^{\circ}$ to the existing beamline, illuminating the ML mirror at a $45^{\circ}$ angle to the X-ray source and the output port. A rotatable flange connects the PSML chamber's output port to the vacuum pipe so that the polarization vector can be rotated through an angle of about $160^{\circ}$. For this phase, the collimator plate is set to an $25 \mathrm{~mm}$ square aperture, which provides a $50 \mathrm{~mm}$ wide illumination pattern at the detector. A front-side illuminated CCD spare from the Suzaku project ${ }^{7}$ was installed on the output port of the detector chamber (see Fig. 1). The CCD has $1024^{2} 24 \mu$ square pixels and is read out by prototype electronics connected to a Sun workstation. See Murphy et al. (2010 $0^{5}$ ) for early details and pictures of the soft X-ray polarization laboratory. The schematic is shown in Fig. 2

The bulk of the work in Phase I was in stabilizing the PSML chamber and aligning the beam so that there would be minimal variation of the count rate in the detector. Fig. 1 1 shows the mirror chamber before a hold-down bracket was installed on the left support bracket in order to prevent lifting of the chamber as the source was rotated about the beamline. The mounts on both sides are secured to the table, which is epoxied to the floor. The ML mirror mount has adjustment screws for two axes of rotation and is attached to the shaft of a rotatable manipulator via a small bracket. Upon stabilizing the system, measurements were consistently repeatable as the source was rotated. 


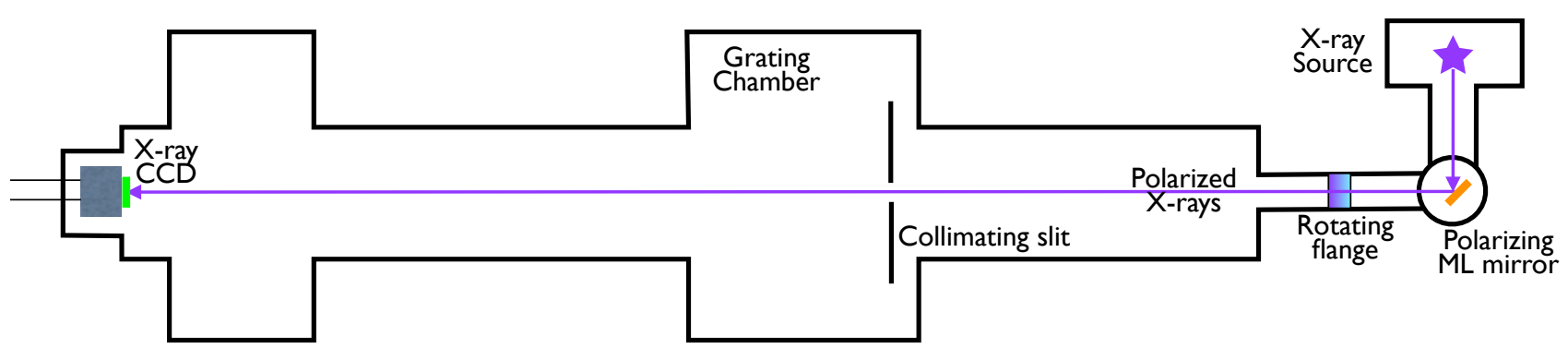

Figure 2. Schematic of the polarimetry beamline in Phase I. The polarization E-vector is oriented perpendicular to the plane of the drawing, for this orientation of the rotating flange. The equipment to the right of the flange is rotated about the beamline axis under computer control by a motor that can also hold the source in place at a range of angles from $-35^{\circ}$ to $+130^{\circ}$, where angles are defined using the right-hand rule pointing to the detector. The diagram shows the configuration at an angle of $+90^{\circ}$. The collimating slit is a $25 \mathrm{~mm}$ square aperture in this phase.
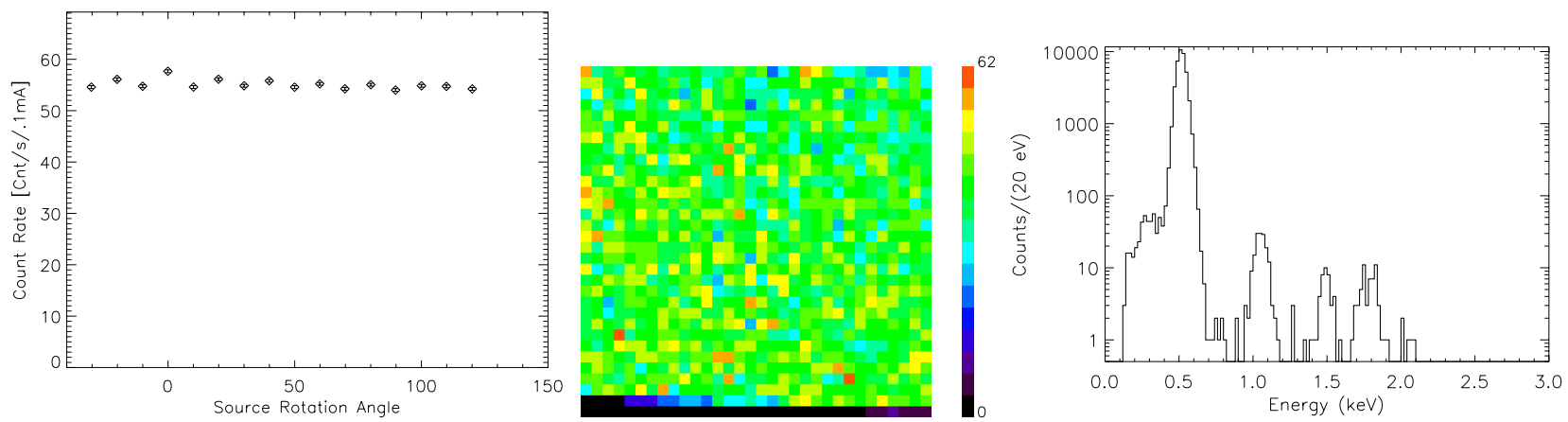

Figure 3. Results from Phase I operations of the polarimetry beamline, where the CCD receives the polarized beam directly (see Fig. 2). Left: The CCD count rate per $0.1 \mathrm{~mA}$ as a function of polarization rotation angle. The source was operated at a voltage of $5 \mathrm{kV}$ and a beam current of $0.3 \mathrm{~mA}$, for $240 \mathrm{~s}$ exposures on 16 July 2013. The beam is uniform with system rotation to better than $5 \%$. Center: CCD image of one of the observations, taken at a rotation angle of $50^{\circ}$. The variation of the counts in $32 \times 32$ pixel regions is only $5 \%$ greater than expected from Poisson counting statistics, indicating that the beam is uniform over scales from $0.8 \mathrm{~mm}$ to $2.5 \mathrm{~cm}$. Right: Pulse distribution from the observation at $50^{\circ}$. Over $95 \%$ of all events are in the O-K peak, as expected. The other features are a tail due to hot pixels below 0.4 $\mathrm{keV}$ and fluorescence from elements in the multilayer, its substrate, and the mirror holder.

Alignment was achieved by setting up a laser in the detector chamber about $16 \mathrm{~m}$ from the X-ray source and pointed through the square collimating aperture to the polarizing mirror, thus defining the beamline optical axis. The mirror readily reflects laser light, so it was rotated to be perpendicular to the laser beam, in order to reflect the laser beam back to the collimating aperture. The mirror chamber mounts (see Fig. 1, left) were adjusted vertically and horizontally and the mirror mount was adjusted to ensure that the reflected laser beam was centered on the collimating aperture, even as the mirror chamber was rotated. In this manner, the mirror's axis was aligned to the beamline axis to within $1 \mathrm{~cm}$ along an $8.5 \mathrm{~m}$ length, for an accuracy of about \pm 0.001 radians.

The rotation angle of the mirror about the manipulator's shaft was set using an alignment laser installed near the source, downstream of the rotating flange. This laser is oriented perpendicular to the beamline, illuminating an insertable prism that provides oppositely directed output beams. The direction of the beam along the beamline is controlled with a micrometer to pass through the collimating aperture in the grating chamber and illuminate the CCD in the detector chamber. The opposite beam reflects off of the source ML mirror to place a small spot on the X-ray source anode, viewable through a viewport at the source. The source anode used for these tests consisted of a $\mathrm{Cu}$ anode with a small sapphire $\left(\mathrm{Al}_{2} \mathrm{O}_{3}\right)$ disk glued into the center of the target face. The anode is oriented at $45^{\circ}$ to both the output and view ports, so the laser light reflects off of the sapphire disk and can be viewed on a translucent screen attached to the viewport. Marking the screen ensures that the mirror orientation can be recovered upon disassembly and subsequent reassembly. 


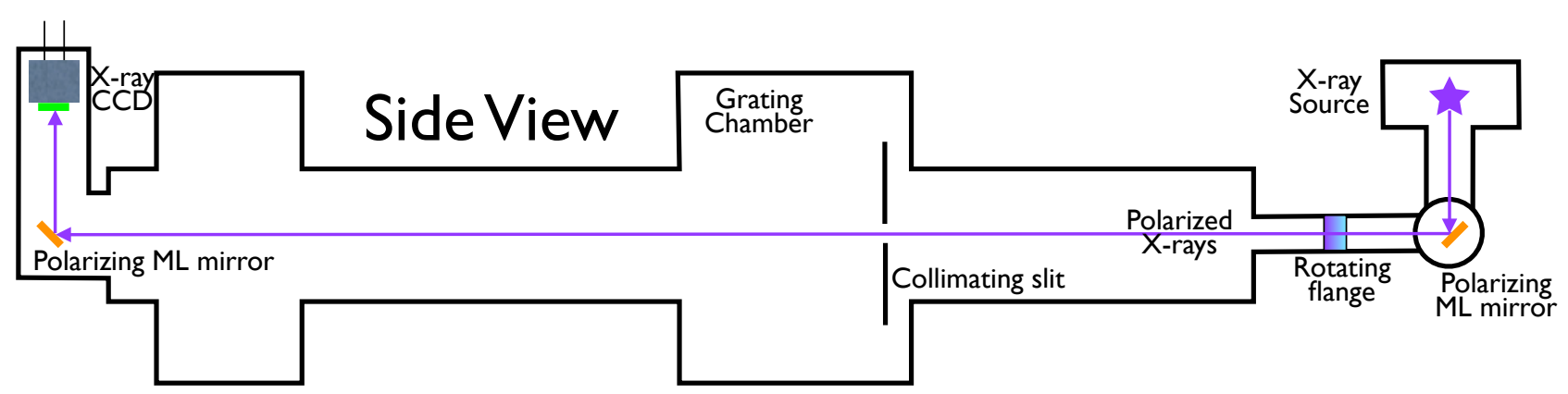

Figure 4. Schematic of the polarimetry beamline in Phase II. The configuration is the same as in Phase I (Fig. 2) except that a polarizing ML mirror has been added to detector end of the beamline and the CCD is reoriented to face it.

Figure 3 shows results from tests after alignment. The source was operated at a voltage of $5 \mathrm{kV}$ and a beam current of $0.3 \mathrm{~mA}$, for $240 \mathrm{~s}$ exposures on 16 July 2013. Measurements were obtained at $20^{\circ}$ steps rotating the system in one direction, then shifting $10^{\circ}$ and stepping $20^{\circ}$ in the reverse direction. The count rate is consistent to within $5 \%$ with rotation of the source and mirror chamber, as shown in the left panel. The middle panel shows that the CCD image is uniform to within $5 \%$ on small scales. The right panel shows an example of the pulse height distribution measured by the CCD for one exposure. Over $95 \%$ of all events are in the O-K peak, as expected. The other features are a tail due to hot pixels below $0.4 \mathrm{keV}$ combined with B-K and C-K fluorescence photons, pulse pileup from two events in a single frame at $1.05 \mathrm{keV}, \mathrm{Al}-\mathrm{K}$ fluorescence near $1.5 \mathrm{keV}$, and $\mathrm{W}-\mathrm{M}$ and $\mathrm{Si}-\mathrm{K}$ fluorescence near $1.8 \mathrm{keV}$. Fluorescence can result from bremsstrahlung photons from the source penetrating the ML coating (comprised of $\mathrm{C}, \mathrm{B}$, and $\mathrm{W}$ ) to the substrate (containing $\mathrm{Si}$ ) and illuminating the $\mathrm{Al}$ mirror holder.

\subsection{Polarimetry Beamline Phase II}

The objective of phase II was to demonstrate that source-mirror combination produces $100 \%$ polarized X-rays. The schematic is shown in Fig. 4 and is the same as for Phase I (Fig. 22) except for the addition of an elbow pipe in which a ML coated mirror is installed and oriented at $45^{\circ}$ to the beam. The mirror is a twin of the source mirror, so it is designed to reflect O-K photons with s-polarization. In the schematic, only those photons with polarizations perpendicular to the plane of the drawing will be reflected. Fig. 5 shows pictures of the added hardware.

As in Phase II, substantial care was taken in mounting and aligning the ML mirror. Alignment was achieved using the system alignment laser mounted in the source end of the system. When reflected off of the mirror, the beam was centered in the vertical pipe along the plane perpendicular to the beam. We also verified that the reflected laser spot illuminated the CCD after it was attached to the vertical pipe by using a camera held in the elbow pipe.

Figure 6 shows results from two different runs in this configuration. In the first one (left panel), only 160 $\mathrm{s}$ exposures were obtained at each angle. The X-ray source was operated at a voltage of $5 \mathrm{kV}$ and $0.3 \mathrm{~mA}$, as was used to obtain the uniformity results shown in Fig. 3. The background was not well determined for the first observation. For the second run, the source voltage was raised to $8 \mathrm{kV}$ and the current raised to $0.5 \mathrm{~mA}$ in order to achieve near maximal source emission. The background was very small, so the modulation factor is consistent with the expectation that the beam is $100 \%$ linearly polarized. As expected, the count rate null is found near $0^{\circ}$ and the maximum is at $90^{\circ}$. The rates are repeatable to within the counting statistics of the measurements, even when several thousand counts were obtained.

Images from these tests showed that the mirror did not reflect all incident photons to the detector. Figure 7 shows that the pattern of illumination on the CCD varied with mirror rotation angle. The pattern is caused by a combination of a nearly monochromatic input beam, a narrow reflectivity curve, and a slight variation of the ML period across the mirror. The mirror, of diameter $d=25 \mathrm{~mm}$ at distance $L$ from the source ML coated mirror, subtends an angle of $\Delta \theta=d / L=0.0015 \mathrm{rad}$ as seen by the source mirror. Differentiating the Bragg condition gives the spread of the peak wavelength, $\Delta \lambda=2 D \cos \theta \Delta \theta$, so the beam's spectral resolution 

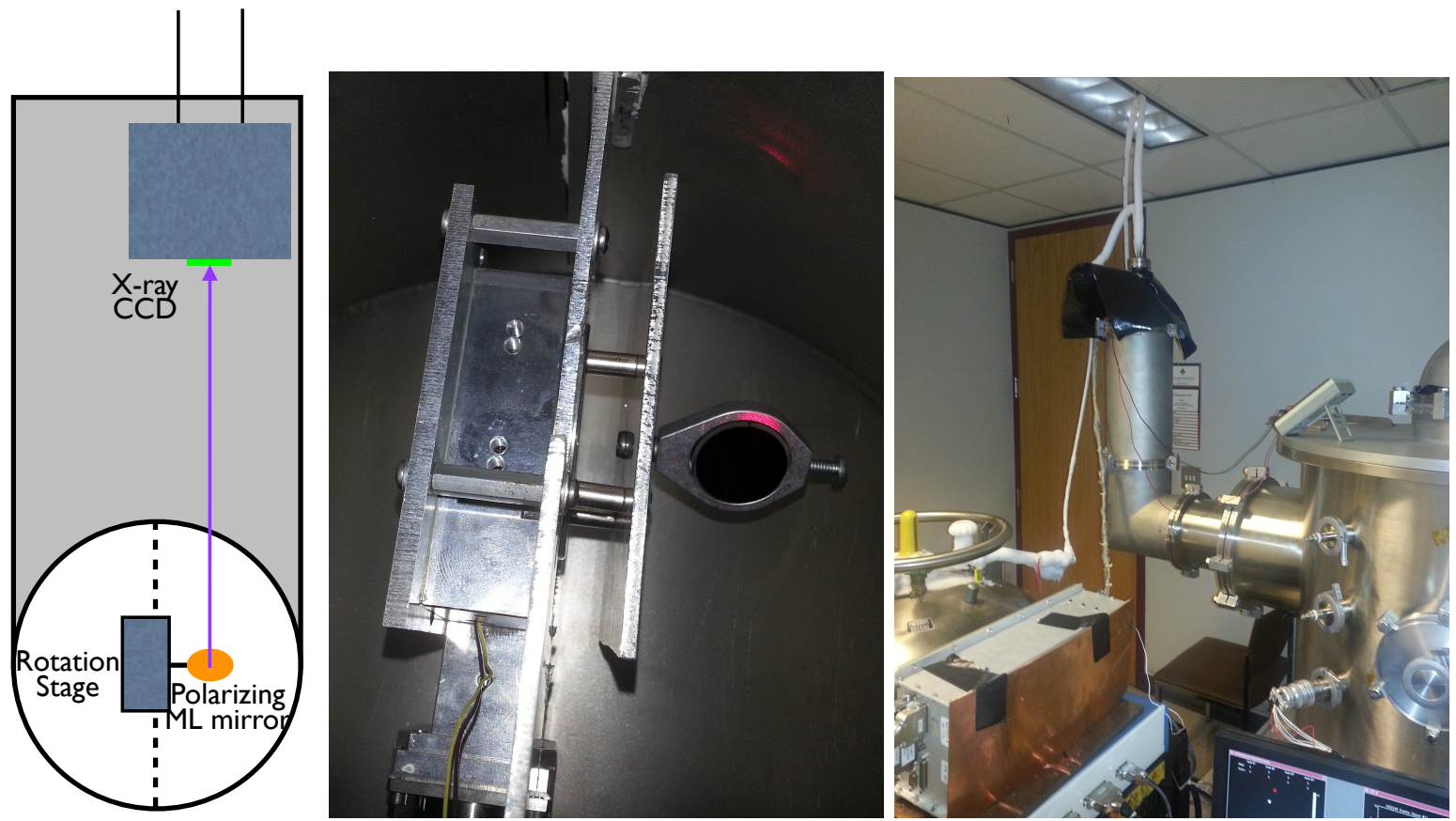

Figure 5. Hardware added to the polarimetry beamline for Phase II. Left: Schematic of the added equipment, as seen from the source along the beamline. Middle: A single-period ML coated mirror is mounted at $45^{\circ}$ to the beamline (from below), as viewed from the flange to which the detector is mounted. The mirror is a twin of the source mirror (see Fig. 1 . left) and is mounted to a rotational stage to allow rotation about the mount axis. The source alignment laser, shown illuminating part of the mirror mount, is used to position and align the mirror. Right: Exterior view of the revised detector end of the system. The ML mirror is mounted in the elbow pipe and reflects X-rays to the detector mounted vertically at the top of the $200 \mathrm{~mm}$ diameter extension pipe. Also visible and used in Phase I are the detector chamber, liquid $\mathrm{N}_{2}$ tank and frosted supply lines, the wrapped data and command wire bundle, a multimeter connected to a resistor on the CCD mount plate to measure its temperature, the CCD electronics boxes, and the screen of the computer that receives data from and sends commands to the CCD.
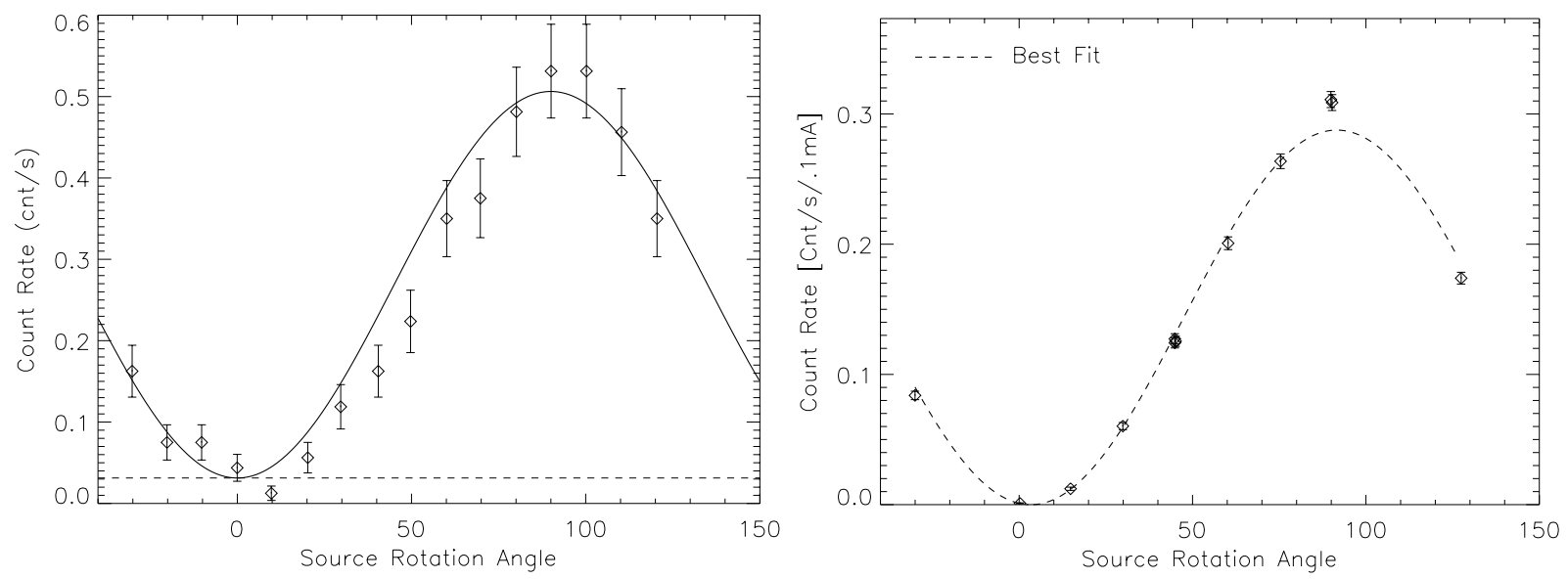

Figure 6. Results from Phase II operations of the polarimetry beamline, where the detector polarizing mirror is installed and the detector is $90^{\circ}$ to the beamline. (see Fig. 4). Left: The source was operated at a voltage of $5 \mathrm{kV}$ and a beam current of $0.3 \mathrm{~mA}$ for $160 \mathrm{~s}$ exposures on 3 August 2013. The dashed line indicates the maximum possible dark contribution. Right: The source was operated at a voltage of $8 \mathrm{kV}$ and a beam current of $0.5 \mathrm{~mA}$ for $1600 \mathrm{~s}$ exposures on 5 August 2013. The dark current is negligible, so the polarization model, a simple sine wave, is an excellent fit to the data. The phase angle of the minimum is consistent with $0^{\circ}$, as expected. 

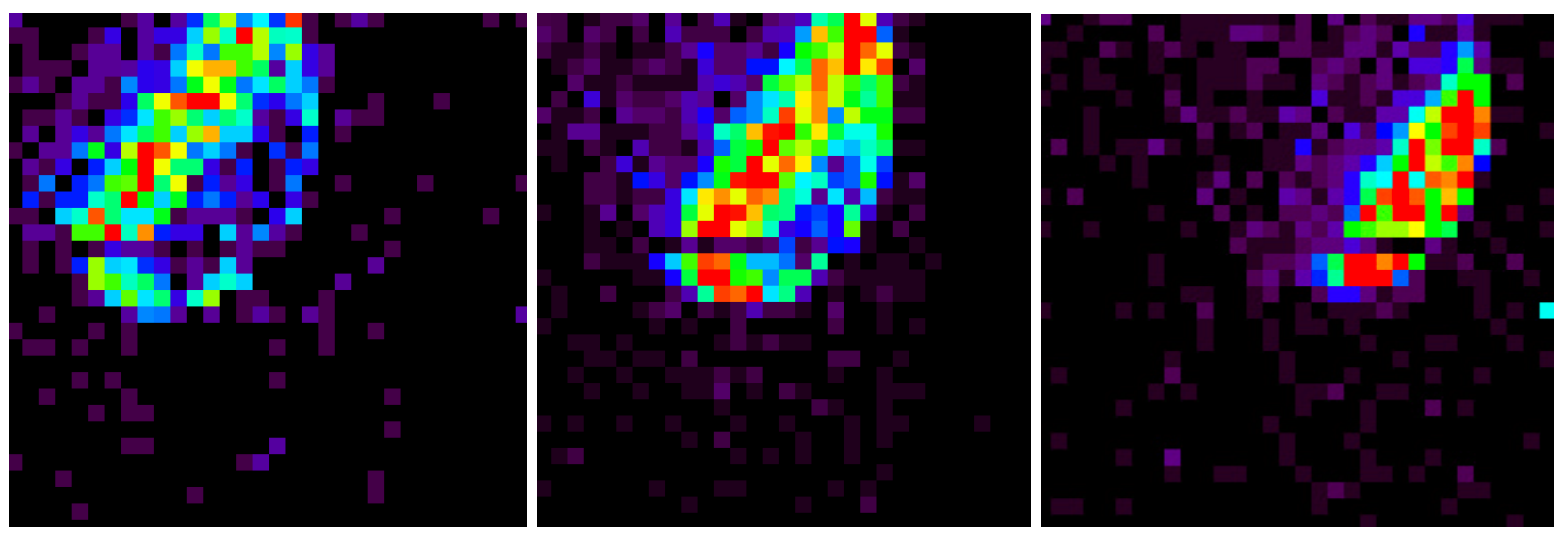

Figure 7. CCD images from Phase II operations of the polarimetry beamline. The $x$ coordinate increases in the direction of the X-ray source. The mirror and CCD are both $25 \mathrm{~cm}$ across but the mirror is tilted at $45^{\circ}$ to the normal of the CCD, so the image from a fully illuminated mirror would be $70 \%$ of the width of the CCD. The detector polarizer mirror was rotated through a range of $0.6^{\circ}$ across the set of observations, both moving the image slightly and changing the image's beam pattern. We deduce that the Bragg peak varies across the surface of the mirror by $1.0 \%$ from center to edge.

is $\Delta \lambda / \lambda=\tan \theta \Delta \theta=\Delta \theta$ for $\theta \approx 45^{\circ}$. Thus, the spectral width of the incident beam in our setup is about $\Delta E=0.0015 E_{\mathrm{O}-\mathrm{K}}=0.8 \mathrm{eV}$. The ML reflectivity curve is about $3 \mathrm{eV} \mathrm{FWHM,} \mathrm{so} \mathrm{the} \mathrm{input} \mathrm{beam} \mathrm{samples} \mathrm{a} \mathrm{small}$ range of the reflectivity curve. When $D$ varies by $1 \%$, the Bragg peak changes by $5 \mathrm{eV}$, where the reflectivity is less than $10 \%$ of the peak. In order to test this possible cause of the nonuniformity, the ML mirror was rotated through an angle of $\Delta \theta=0.01 \mathrm{rad}=0.6^{\circ}$. Fig. 7 shows that the this rotation was sufficient to move off of the Bragg peak at the center of the mirror and to the Bragg peak at the edge, indicating that there is a $1 \%$ gradient of the ML $D$ spacing from center to edge, consistent with the manufacturing tolerance.

\subsection{Polarimetry Beamline Phase III}

With funding from a MIT Kavli Investment grant, we started Phase III, where the goal is to develop, test, and install a laterally graded ML coated mirror (LGML) in the source mirror chamber. A pair of LGMLs were fabricated by RXO, consisting of 200 bilayers of $\mathrm{W}$ and $\mathrm{B}_{4} \mathrm{C}$ on highly polished Si wafers. The $D$ spacing was varied in order to reflect and polarize X-rays from $17 \AA$ to $73 \AA$ (170 to $730 \mathrm{eV})$. Fig. 8 shows a picture of one such LGML and the results from a reflectivity run at the ALS.

We will soon reconfigure the source ML mirror chamber by replacing the current single-period ML mirror with a LGML. We have obtained a motor-controlled rotational manipulator and will mount the LGML to its shaft so that the axis of the shaft is centered on the long axis of the LGML's surface. The shaft will be controllable to $0.01^{\circ}$ and will be mounted on a linear bellows with a $100 \mathrm{~mm}$ travel that is computer-controlled to an accuracy of $0.01 \mathrm{~mm}$. After alignment, we will calibrate the linear stage to determine the insertion points that maximize reflectivity at a wide variety of soft X-ray emission lines from different anodes used in the X-ray source. For this phase, the CCD will be returned to the position it had in Phase I. Upon completion of Phase III, the X-ray source will be capable of generating $100 \%$ polarized X-rays at a wide range of energies and rotating the polarization direction through at least $150^{\circ}$.

\subsection{Polarimetry Beamline Phase IV}

With new funding from the NASA Astrophysics Research and Analysis (APRA) program, we will move to Phase IV, where the goals are 1) to improve the reflectivities of LGMLs by trying new material combinations and 2) show that a grating-LGML combination can measure polarization over wide range of energies, thus prototyping a design that could be used for a flight system. See Fig. 9 for a schematic of the Phase IV configuration.

The first improvement to the LGML reflectivities will involve using different ML compositions for specific wavelength regions. For example, MLs with $\mathrm{C} / \mathrm{Cr}$ bilayers are known to perform better than $\mathrm{W} / \mathrm{B}_{4} \mathrm{C}$ MLs. Figure 10 shows results from ALS testing of the first few single-period MLs involving $\mathrm{C}$ and $\mathrm{Cr}$ or a $\mathrm{CoCr}$ alloy. The $\mathrm{C} / \mathrm{CoCr}$ MLs are slightly better and the predicted reflectivities to s-polarization approach 20\% in the 45-65 

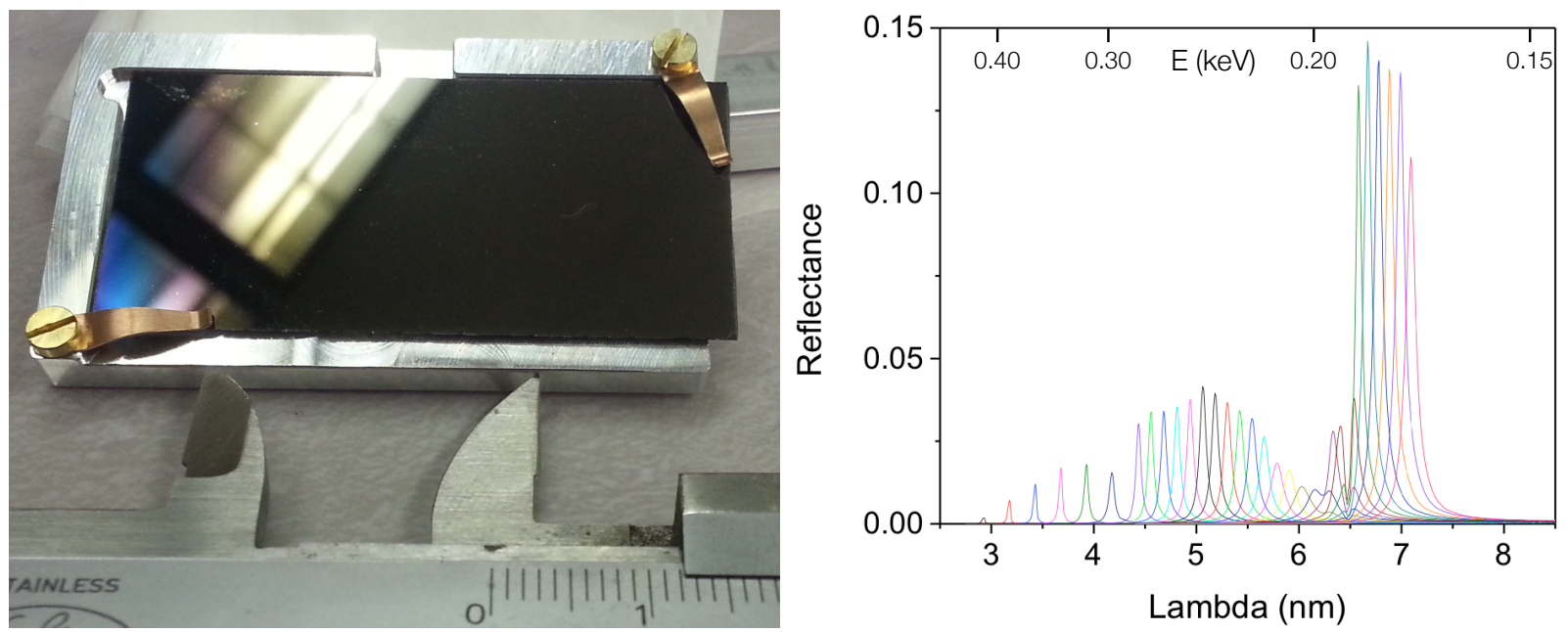

Figure 8. Left: A laterally graded ML coated mirror (LGML) from RXO in a holder. It is about $47 \mathrm{~mm}$ long, $23 \mathrm{~mm}$ wide, and $0.5 \mathrm{~mm}$ thick. The $D$ spacing increases from left to right. Right: Reflectivity measurements of a LGML from RXO. For one measurement run, the reflectivity was sampled a $2 \mathrm{~mm}$ spacing, starting below $3 \mathrm{~nm}$. In a second run, the samples were at $1 \mathrm{~mm}$ spacing. The ALS beam consisted of about $70 \%$ s-polarization and data were taken at a graze angle of $45^{\circ}$.
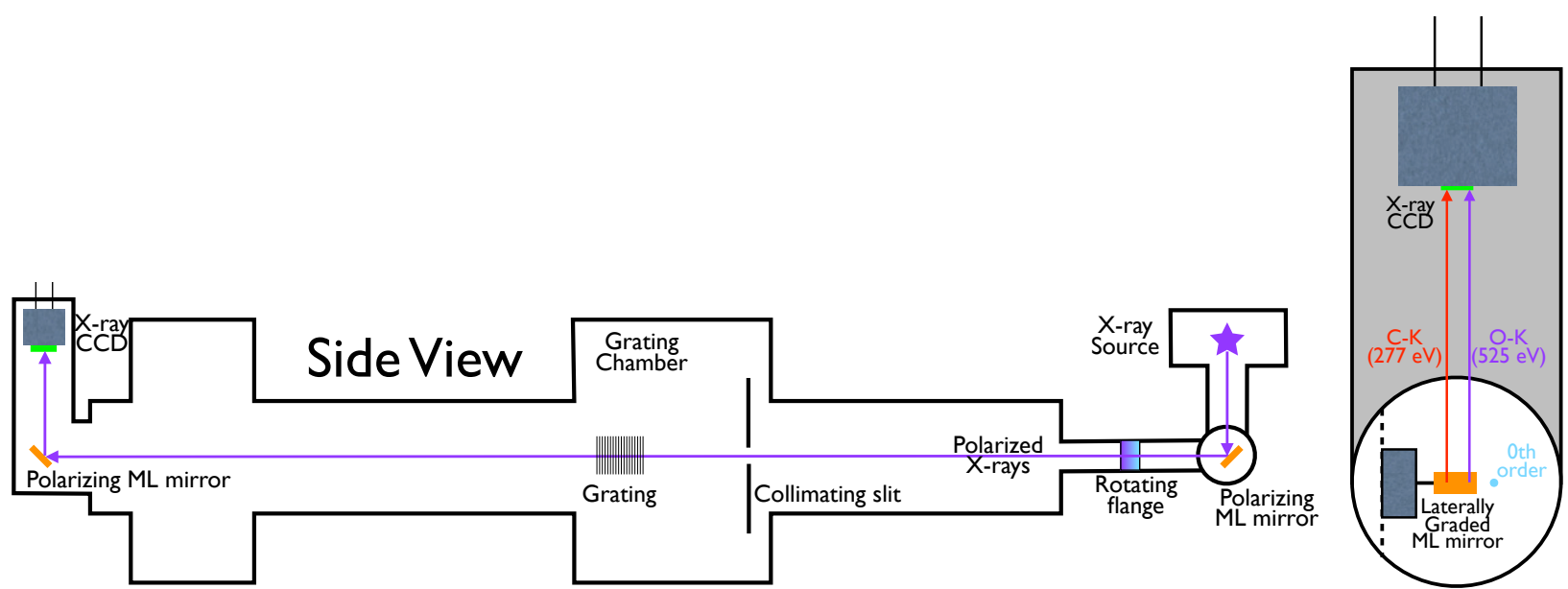

Figure 9. Schematic of the polarimetry beamline in Phase IV. The configuration is the same as in Phase II (Fig. 4 ) except that the polarizing ML mirrors at both ends of the system are replaced with LGMLs and there is a grating mounted in the grating chamber to disperse X-rays to specific locations on the detector LGML. Left: Side view, showing the location of the grating. Right: Beam view, showing that the LGML will reflect different input energies from different locations on its surface. 

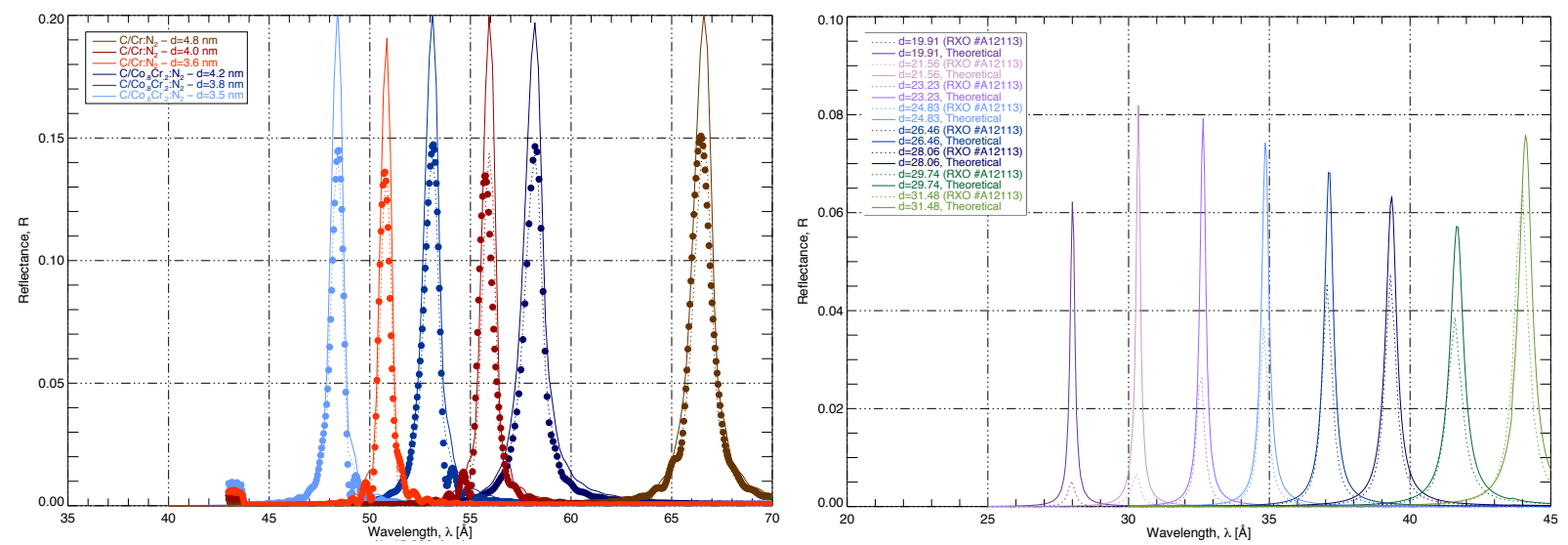

Figure 10. Multilayer reflectivities for single-period coatings. New laterally graded ML mirrors will be developed using these coating combinations. Left: Several examples of new single-period multilayer coating samples that include C for use in the 44-65 $\AA$ band. Data are shown with model fits (dotted lines) from the partially polarized ALS measurements. Solid lines show reflectivity for the same models but for $100 \%$ polarized X-rays, which indicate that the s-polarization reflectivities approach $20 \%$ in this wavelength range. Right: Measurements from a Laterally graded ML coated sample from RXO are shown with dotted lines compared to theoretically achievable reflectivities. The coating was made by sputtering 200 bilayers of $\mathrm{W}$ and $\mathrm{B}_{4} \mathrm{C}$ with $\mathrm{N}_{2}$ in the chamber. By varying the deposition approach, we expect to improve reflectivities to approach the theoretical values.

$\AA$ range. These reflectivites are substantially better than the $2-5 \%$ values currently available in the $\mathrm{W} / \mathrm{B}_{4} \mathrm{C}$ LGMLs (Fig. 8, right). ML coating process adjustments will be varied in order to improve the reflectivities below $45 \AA$.

The second advance in Phase IV will be to use gratings to disperse the X-rays to the LGML in the detector chamber. The dispersion of the grating is given by the grating equation: $m \lambda=P \sin \phi$, where $P$ is the grating period, $m$ is the grating order of interest (which we take to be +1 ) and $\phi$ is the dispersion angle. Defining $y$ to be the horizontal direction in Fig. 9 right and defining $y=0$ to be where the 0th order lands at the plane of the LGML, then we match the LGML's Bragg peak to the grating dispersion by setting $P \sin \phi=P y / D_{g}=$ $2 D(y) \sin \theta=\sqrt{ } 2 D(y)$, where $D_{g}$ is the distance from the grating to the LGML, giving $D=P y /\left(D_{g} \sqrt{ } 2\right)$. As long as $D(y)$ is linear, the LGML can be placed at the appropriate distance, $D_{g}$, from the grating to reflect X-rays of arbitrary wavelengths, within the physical limitation of the LGML. The current LGMLs have $D$ gradients of $0.87 \AA / \mathrm{mm}$, which is matchable by gratings made for the Chandra Low Energy Transmission Grating (LETG) Spectrometer ${ }^{8}$ for $D_{g}=8.7 \mathrm{~m}$. We have four LETG facets on loan from MPE (courtesy P. Predehl) that will be mounted in the grating chamber for this purpose.

\section{A SOFT X-RAY POLARIZING SPECTROMETER}

The basic design of a polarizing spectrometer was outlined by Marshall $\left(2008^{2}\right)$. For this paper, we examine the approach that could be applied to a suborbital rocket experiment. Figure 11 shows a possible schematic for a suborbital mission using blazed gratings such as the Critical Angle Transmission (CAT) gratings under development at MIT 910 Sampling at least 3 position angles is required in order to measure three Stokes parameters $(\mathrm{I}, \mathrm{Q}, \mathrm{U})$ uniquely, so one would require at least three separate detector systems (one of which could be just for 0th order) with accompanying multilayer-coated flats or that the rocket rotate during the observations (which is expected anyway, to take out systematic effects).

The system design consists of a mirror system with an assumed effective area of $350 \mathrm{~cm}^{2}$ below $1 \mathrm{keV}$, backside-illuminated CCD detectors like those on Chandra with thin directly deposited optical blocking filters, and CAT gratings blazed to maximize efficiency at $300 \mathrm{eV}$. We have also computed effective areas using medium energy gratings (MEGs ${ }^{11}$ ) and LETGs. ${ }^{8}$ For ML coating reflectivities, we used values that have been achieved in the lab for single-period MLs used at $45^{\circ}$ and interpolated using comparable theoretical models. The resulting achievable effective area is shown in Fig. 12 . 


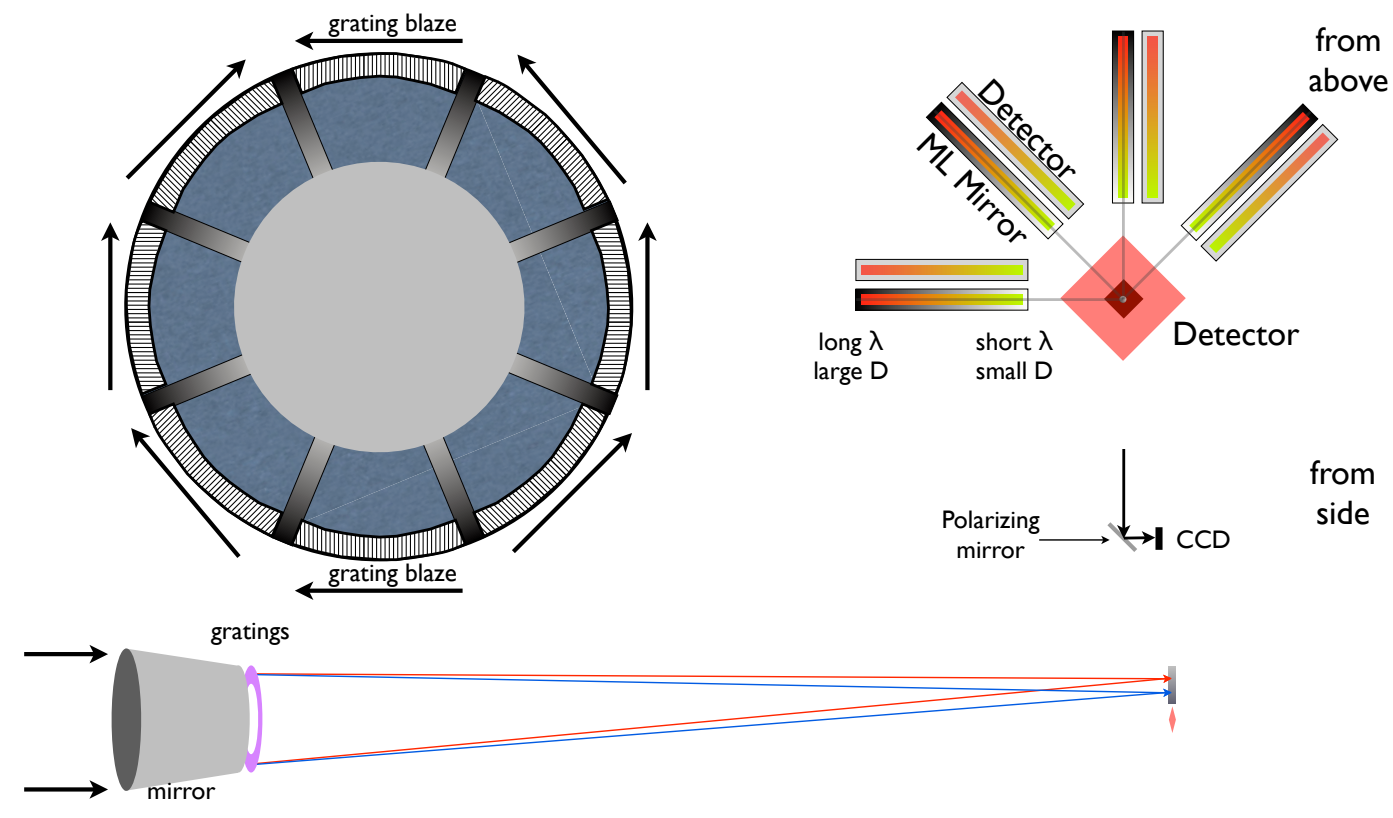

Figure 11. Schematic of a suborbital soft X-ray polarimeter using blazed gratings such as the CAT gratings under development at MIT! Bottom: The target is to the left and the dispersion by one set of gratings is shown. Left: View of the front aperture, where blazed gratings are oriented approximately radially and in sectors to improve resolution along the dispersion direction. Right, above: Top view of a focal plane layout that could be used for a suborbital rocket experiment, in the manner suggested by Marshall $\left(2008^{2}\right)$. The zeroth order is placed at the location of the gray dot so that the dispersed spectrum first intercepts the laterally graded multilayer mirror that is angled at $45^{\circ}$ to the incoming X-rays. Right, below: Side view, where the dispersion is perpendicular to the plane of the drawing and the multilayer mirror is oriented $45^{\circ}$ to the incoming, dispersed X-rays.
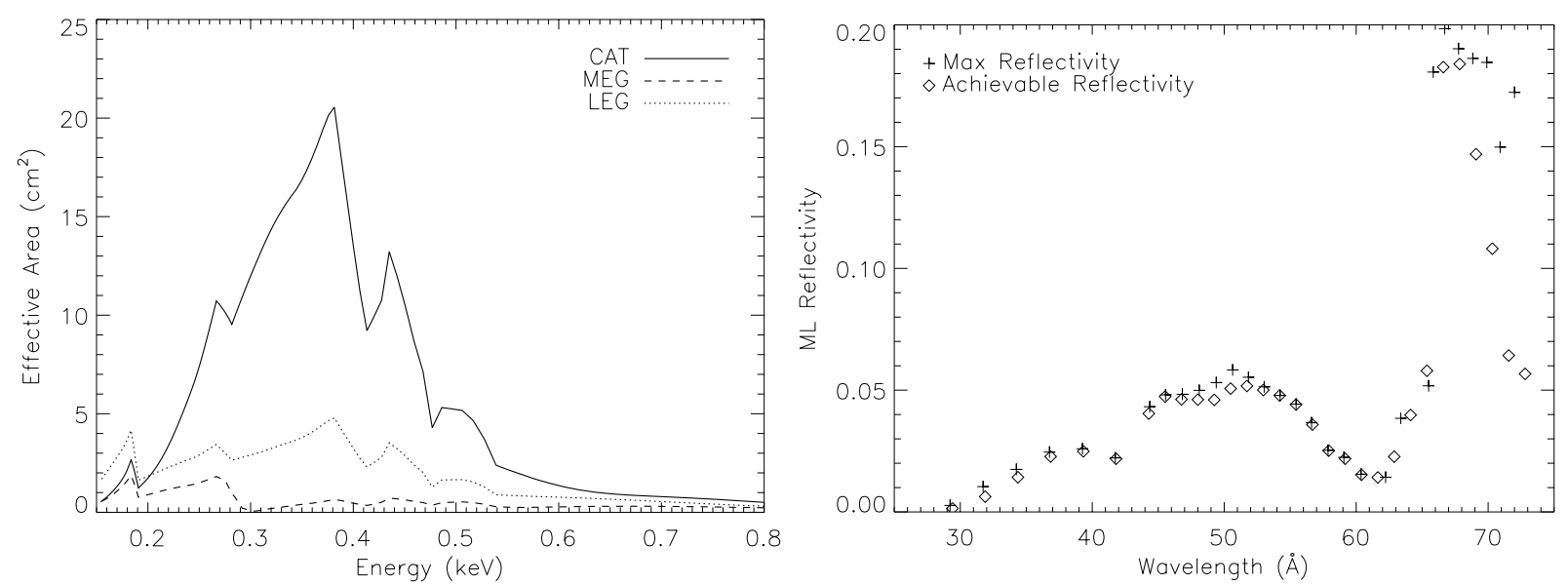

Figure 12. Left: Effective area of a suborbital polarimeter mission using achievable ML reflectivities. Right: Maximum reflectivities at various locations on a LGML from RXO (plus signs), compared to values obtained using a strictly linear dispersion (diamonds). 
The effective area estimate can be used to predict the minimum detectable polarization (MDP) for a potential target. Extragalactic sources such as the BL Lac object Mk 421 are expected to be highly polarized in the soft X-ray band. Mk 421 is currently the brightest BL Lac object, and a recent Chandra LETG spectrum was readily fit by a power law spectrum $\left(n_{E}=n_{0} E^{-\Gamma} e^{-N_{H} \sigma[E]}\right.$, where $\sigma[E]$ is the energy dependent atomic cross section of the interstellar medium with cosmic abundances and $E$ is in $\mathrm{keV}$ ) with $n_{0}=0.2 \mathrm{ph} \mathrm{cm}^{-2} \mathrm{~s}^{-1} \mathrm{keV}^{-1} \Gamma=2.7$, and an assumed cold column density of $N_{H}=1.61 \times 10^{20} \mathrm{~cm}^{-2}$. In a $500 \mathrm{~s}$ observation of Mk 421, this instrument could detect polarizations of $3.9 \%$ using CAT gratings, $11.5 \%$ using MEGs, or $6.5 \%$ using LETGs.

LGMLs with the achievable reflectivities have not yet been fabricated, so we recomputed the MDPs for reflectivities as measured by the ALS for the LGML made by RXO. Interpolating using the Bragg peak reflectivities at the measured energies gives MDPs of $11.4 \%$ for CAT gratings, and $16 \%$ using LETGs. However, we note that the Bragg peaks do not precisely correspond to the locations of a linearly dispersed spectrum, so that the reflectivity will be somewhat smaller than measured at some wavelengths. See Fig. 12 (right) for estimates of how much the reflectivities will decrease. We have used the observed reflectivity curves to compute that the MDP will increase by about $10 \%$ due to slight nonlinearity of the actual manufactured LGML, so we expect that a suborbital flight using the existing LGMLs would reach $18 \%$ using existing LETGs or improve to $12 \%$ should CAT (or high efficiency reflection) gratings be available. Continued development of LGMLs are expected to bring these MDPs down below $10 \%$.

\section{ACKNOWLEDGMENTS}

We are very grateful for the assistance and support provided by Steve Kissel and Beverly LaMarr in providing, operating, and modifying the CCD detector system. MIT undergraduates Keven Jenks and Kelly Kochanski helped set up and operate the X-ray source. We thank Regina Soufli for suggesting and facilitating the ALS measurements of the laterally graded multilayer coatings. Support for this work was provided by the National Aeronautics and Space Administration through grant NNX12AH12G and by Research Investment Grants from the MIT Kavli Institute.

\section{REFERENCES}

1. H. L. Marshall, "A soft x-ray polarimeter designed for broadband x-ray telescopes," in Optics for EUV, X-Ray, and Gamma-Ray Astronomy III. Edited by O'Dell, Stephen L.; Pareschi, Giovanni. Proceedings of the SPIE, Volume 6688, pp. $66880 Z$ (2007)., Presented at the Society of Photo-Optical Instrumentation Engineers (SPIE) Conference 6688, Sept. 2007.

2. H. L. Marshall, "Polarimetry with a soft x-ray spectrometer," in Society of Photo-Optical Instrumentation Engineers (SPIE) Conference Series, Society of Photo-Optical Instrumentation Engineers (SPIE) Conference Series 7011, Aug. 2008.

3. H. L. Marshall, S. S. Murray, J. H. Chappell, H. W. Schnopper, E. H. Silver, and M. C. Weisskopf, "Realistic, inexpensive, soft x-ray polarimeter and the potential scientific return," in Polarimetry in Astronomy. Edited by Silvano Fineschi . Proceedings of the SPIE, Volume 4843, pp. 360-371 (2003)., S. Fineschi, ed., Presented at the Society of Photo-Optical Instrumentation Engineers (SPIE) Conference 4843, pp. 360-371, Feb. 2003.

4. H. L. Marshall, R. K. Heilmann, N. S. Schulz, and K. D. Murphy, "Broadband soft x-ray polarimetry," in Society of Photo-Optical Instrumentation Engineers (SPIE) Conference Series, Society of Photo-Optical Instrumentation Engineers (SPIE) Conference Series 7732, July 2010.

5. K. D. Murphy, H. L. Marshall, N. S. Schulz, K. P. Jenks, S. J. B. Sommer, and E. A. Marshall, "Soft x-ray polarimeter laboratory tests," in Society of Photo-Optical Instrumentation Engineers (SPIE) Conference Series, Society of Photo-Optical Instrumentation Engineers (SPIE) Conference Series 7732, June 2010.

6. D. Dewey, D. N. Humphries, G. Y. McLean, and D. A. Moschella, "Laboratory calibration of x-ray transmission diffraction gratings," in Proc. SPIE Vol. 2280, p. 257-271, EUV, X-Ray, and Gamma-Ray Instrumentation for Astronomy V, Oswald H. Siegmund; John V. Vallerga; Eds., O. H. Siegmund and J. V. Vallerga, eds., Presented at the Society of Photo-Optical Instrumentation Engineers (SPIE) Conference 2280, pp. 257-271, Sept. 1994. 
7. B. LaMarr, M. W. Bautz, S. E. Kissel, G. Y. Prigozhin, K. Hayashida, T. G. Tsuru, and H. Matsumoto, "Ground calibration of X-ray CCD detectors with charge injection for the X-ray imaging spectrometer on Astro-E2," in Society of Photo-Optical Instrumentation Engineers (SPIE) Conference Series, A. D. Holland, ed., Society of Photo-Optical Instrumentation Engineers (SPIE) Conference Series 5501, pp. 385-391, Sept. 2004.

8. P. Predehl, H. W. Braeuninger, A. C. Brinkman, D. Dewey, J. J. Drake, K. A. Flanagan, T. Gunsing, G. D. Hartner, J. Z. Juda, M. Juda, J. S. Kaastra, H. L. Marshall, and D. A. Swartz, "X-ray calibration of the AXAF Low Energy Transmission Grating Spectrometer: effective area," in Society of Photo-Optical Instrumentation Engineers (SPIE) Conference Series, R. B. Hoover and A. B. Walker, eds., Society of Photo-Optical Instrumentation Engineers (SPIE) Conference Series 3113, pp. 172-180, July 1997.

9. R. K. Heilmann, M. Ahn, E. M. Gullikson, and M. L. Schattenburg, "Blazed high-efficiency x-ray diffraction via transmission through arrays of nanometer-scale mirrors," Opt. Express 16(12), pp. 8658-8669, 2008.

10. R. K. Heilmann, M. Ahn, M. W. Bautz, R. Foster, D. P. Huenemoerder, H. L. Marshall, P. Mukherjee, M. L. Schattenburg, N. S. Schulz, and M. Smith, "Development of a critical-angle transmission grating spectrometer for the International X-Ray Observatory," in Society of Photo-Optical Instrumentation Engineers (SPIE) Conference Series, Society of Photo-Optical Instrumentation Engineers (SPIE) Conference Series 7437, Aug. 2009.

11. C. R. Canizares, J. E. Davis, D. Dewey, K. A. Flanagan, E. B. Galton, D. P. Huenemoerder, K. Ishibashi, T. H. Markert, H. L. Marshall, M. McGuirk, M. L. Schattenburg, N. S. Schulz, H. I. Smith, and M. Wise, "The Chandra High-Energy Transmission Grating: Design, Fabrication, Ground Calibration, and 5 Years in Flight," PASP 117, pp. 1144-1171, Oct. 2005. 